\title{
Influence of low-level laser therapy on the healing of human bone maxillofacial defects: A systematic review
}

\author{
Carolina dos Santos Santinoni ${ }^{\mathrm{a}, \mathrm{b}}$, Hiskell Francine Fernandes Oliveira ${ }^{\mathrm{a}}$, Victor Eduardo de Souza Batista ${ }^{\mathrm{a}}$, \\ Cleidiel Aparecido Araujo Lemos ${ }^{\text {a }}$, Fellippo Ramos Verri ${ }^{\text {a,* }}$ \\ a Dental School, Univ. Estadual Paulista - UNESP, Araçatuba, Brazil \\ b Dental School, Univ. do Oeste Paulista - UNOESTE, Presidente Prudente, Brazil
}

\section{A R T I C L E I N F O}

\section{Article history:}

Received 29 October 2016

Received in revised form 4 March 2017

Accepted 6 March 2017

Available online 7 March 2017

\section{Keywords:}

Low-level laser therapy

Lasers

Bone regeneration

Bone

Dentistry

Maxillofacial

Systematic review

Review

\begin{abstract}
A B S T R A C T
Purpose: This systematic review evaluates the effectiveness of low-level laser therapy (LLLT) to enhance maxillofacial area bone repair.

Methods: A comprehensive search of studies published up to February 2017 and listed in PubMed/MEDLINE, Scopus, and Cochrane Library databases was performed in accordance with the Preferred Reporting Items for Systematic Reviews and Meta-Analyses (PRISMA) statement.

Results: The 15 selected studies evaluated a total of 374 patients (mean age, 28.5 years) who were treated with LLLT. Gallium-arsenide (GaAs) and gallium aluminium arsenide (GaAlAs) were the most commonly used devices, and LLLT parameters varied greatly. Wavelengths varied from 500 to $1000 \mathrm{~nm}$. Tooth extraction, distraction osteogenesis, maxillary expansion, periodontal defects, orthodontic movement and maxillary cystic defects were evaluated. From the 15 selected studies, six evaluated bone repair (primary outcomes). Of these, four studies showed improvement in bone formation after using LLLT, two demonstrated improved results for only one follow up period, and one showed no additional benefits. The other 9 studies evaluated secondary parameters related to healing (secondary outcomes) in the maxillofacial area after applying LLLT, including antiinflammatory, analgesic, and healing accelerator effects, and quality of life related to oral health. There were no adverse or negative effects of LLLT reported.

Conclusion: Within the limitation of this review, a possible improvement in bone density can be found when LLLT is applied postoperatively in maxillofacial bony defects. LLLT also seems to promote anti-inflammatory and analgesic effects and accelerate healing, as well as enhance quality of life related to oral health. However, LLLT use protocols need to be standardized before more specific conclusions can be drawn about this subject.
\end{abstract}

(c) 2017 Elsevier B.V. All rights reserved.

\section{Introduction}

The application of lasers has been considered a technological advance. They are used as an adjuvant because of their therapeutic effect and to biostimulate tissues. Low-level lasers, applied in the red spectral region and near infrared regions, promote cellular photobiomodulation effects and therapeutic responses induced by photochemical, photoelectric, and photoenergetic reactions (1).

Low-level laser therapy (LLLT) has been used by researchers in several health fields to accelerate wound healing in hard and soft tissues. In Medicine, this and other therapies have been applied for surgical scars [2,3], and for wrist and hand fractures [4]. In Dentistry, laser therapy has been clinically used and evaluated for post-surgical tooth extraction [5-8], after rapid maxillary expansion $[1,9,10]$, after connective tissue graft

\footnotetext{
* Corresponding author at: Depto. Materiais Odontológicos e Prótese, Faculdade de Odontologia de Araçatuba - UNESP, Rua José Bonifácio, 1193, 16015-050 Araçatuba, SP, Brazil.

E-mail address: fellippo@foa.unesp.br (F.R. Verri).
}

harvesting in the mucosal palate [11], for treatment of jaw osteonecrosis $[12,13]$, and for periodontal defects $[14,15]$.

Bone has the capacity to regenerate and repair itself. However, this capacity may be impaired or lost depending on the size of the defect or the presence of certain diseases [16]. Thus, researchers have directed their efforts to find a therapy that can improve its healing ability. In vivo and in vitro studies have demonstrated that LLLT can improve bone healing by activating the osteogenic factors $[17,18]$. In addition, LLLT can also stimulate angiogenesis, a key component of bone formation during the early phase of healing $[19,20]$, and induce cell proliferation [18,21]. Although positive results have been reported from clinical, animal, and in vitro and experiments, other studies that investigated the effects of LLLT on bone healing are contradictory $[22,23]$. Such discrepancies might be attributable to variations in the irradiation protocols and/or the experimental models used [18,24].

The application of LLLT to bone tissue has already been critically evaluated by some authors. Systematic reviews assessed its effect on in vitro proliferation and differentiation of bone cell lines [25], in vivo reduction 
of the duration of osseointegration in dental implants [26], treatment of peri-implantitis [27] or periodontitis [28-30], and accelerating orthodontic tooth movement $[31,32]$. LLLT was also systematically analyzed as a potential approach for management of osteonecrosis of the jaw [33].

Doeuk et al. [34] performed a systematic review to generally evaluate low level laser treatment in maxillofacial surgery in general way. However, there is no systematic review that evaluates the effect of this therapy in promoting the formation of bone tissue. Thus, the aim of this systematic review is to assess the null hypotheses that there are no differences between bone defects in maxillofacial areas treated with lasers compared to a control group. Published scientific studies were reviewed to assess the information available on this topic to provide a more detailed understanding of the clinical effects of LLLT on enhancing bone repair, and any other relevant circumstances regarding healing in Dentistry.

\section{Material and Methods}

This systematic review is based on the Preferred Reporting Items for Systematic Reviews and Meta-Analyses (PRISMA) checklist structure [35] and in accordance with a model proposed in previously published reports [36,37]. Moreover, this study was registered on the international prospective register of systematic reviews (PROSPERO CRD42016041899). Two independent investigators (C.S.S. and H.F.F.O.) conducted an electronic search of PubMed/MEDLINE, Scopus, and Cochrane Library for articles published up to February 2017, using the following search terms: low-level laser therapy AND bone repair OR low-level laser therapy AND bone healing OR low-level laser therapy AND bone regeneration. These researchers manually searched for articles published in the following journals: Laser in Medical Science, Photomomedicine and Laser Surgery, and the Journal of Photochemistry and Photobiology B Biology. They also conducted a search of the nonpeer reviewed reports and currently unpublished registered trials. All differences in choices between the investigators were analyzed by a third investigator (F.R.V.), and consensus was reached through discussion.

Studies were independently selected and classified as included or excluded by the two researchers (C.S.S. and H.F.F.O.), based on the title and abstract of the articles. Eligible studies included randomized controlled trials (RCTs), studies that compared LLLT and other treatments to promote bone formation, studies that had at least 10 participants, and studies published in English. Exclusion criteria were retrospective or prospective studies, in vitro or animal studies, computer simulations, case reports, studies that evaluated only one type of treatment without a comparison group, and published report reviews. A specific question was formulated based on the population, intervention, control, and outcome (PICO) criteria. The focused question was: "Is LLLT effective in promoting bone regeneration in the maxillofacial area?" Based on these criteria, the population was the participants who were treated with low-level laser therapy in the maxillofacial area to promote bone regeneration, the intervention was low-level laser therapy, and the comparison was control groups. The primary outcome was bone formation, and secondary outcomes were anti-inflammatory, analgesic, and healing accelerator effects, as well as quality of life related to oral health.

Data extracted from the articles were sorted as quantitative or qualitative by one of the researchers (C.A.A.L.) and then checked by two others (F.R.V. and V.S.E.B.). Any disagreements were resolved through discussion until consensus was reached. The quantitative and qualitative data were tabulated for ease of comparison (Tables 1,2 and 3).

Two investigators (C.A.A.L. and V.E.S.B.) assessed the methodological quality of studies according to the Jadad scale, which ranges from 0 to 5 , with studies that scored greater than or equal to 3 considered to be high quality [38] (Table 4). The Cochrane collaboration criteria for judging risk of bias were used to assess the quality of the studies included in the review.

The kappa coefficient value was calculated to determine inter-reader agreement in the study selection process for publications in the PubMed/MEDLINE, Scopus, and Cochrane Library databases.

Table 1

Qualitative characteristics of the studies related to patients.

\begin{tabular}{|c|c|c|c|c|c|c|}
\hline Author & Study design & Gender & Systemic conditions & $\begin{array}{l}\text { Sample } \\
\text { size }\end{array}$ & $\begin{array}{l}\text { Mean of age } \\
\text { (years) }\end{array}$ & Study site \\
\hline $\begin{array}{l}\text { Ferreira et al., } \\
2016\end{array}$ & $\begin{array}{l}\text { Groups evaluated in } \\
\text { different subjects }\end{array}$ & $\mathrm{F} / \mathrm{M}$ & Healthy & 14 & 11 & University \\
\hline Zaky et al., 2016 & $\begin{array}{l}\text { Groups evaluated in } \\
\text { different subjects }\end{array}$ & $\begin{array}{l}\text { Not } \\
\text { described }\end{array}$ & $\begin{array}{l}\text { Excluded as study subjects if they have any systemic disease that } \\
\text { interferes with bone healing }\end{array}$ & 16 & 32 & $\begin{array}{l}\text { Research } \\
\text { Center }\end{array}$ \\
\hline $\begin{array}{l}\text { Abd-Elaal et al., } \\
2015\end{array}$ & Split mouth & $\mathrm{F} / \mathrm{M}$ & Healthy & 10 & 31 & Hospital \\
\hline $\begin{array}{l}\text { Domínguez et al., } \\
2015\end{array}$ & Split mouth & $\mathrm{F} / \mathrm{M}$ & Healthy & 10 & 13 & University \\
\hline $\begin{array}{l}\text { Garcia et al., } \\
\quad 2015\end{array}$ & $\begin{array}{l}\text { Groups evaluated in } \\
\text { different subjects }\end{array}$ & $\mathrm{F} / \mathrm{M}$ & Healthy & 39 & 8 & Hospital \\
\hline $\begin{array}{l}\text { Eslamian et al., } \\
2014\end{array}$ & Split mouth & $\mathrm{F} / \mathrm{M}$ & Healthy & 37 & 24 & University \\
\hline $\begin{array}{l}\text { Mozzati et al., } \\
2012\end{array}$ & Split mouth & $\mathrm{F} / \mathrm{M}$ & Patients waiting for liver transplantation & 20 & - & University \\
\hline $\begin{array}{l}\text { Mozzati et al., } \\
2011\end{array}$ & Split mouth & $\mathrm{F} / \mathrm{M}$ & Healthy & 10 & 22,5 & University \\
\hline $\begin{array}{l}\text { Angeletti et al., } \\
2010\end{array}$ & $\begin{array}{l}\text { Groups evaluated in } \\
\text { different subjects }\end{array}$ & $\mathrm{F} / \mathrm{M}$ & Healthy & 13 & 24 & University \\
\hline $\begin{array}{l}\text { AboElsaad et al., } \\
2009\end{array}$ & $\begin{array}{l}\text { Groups evaluated in } \\
\text { different subjects }\end{array}$ & $\mathrm{F} / \mathrm{M}$ & Healthy & 20 & 45 & Hospital \\
\hline $\begin{array}{l}\text { Angelov et al., } \\
2009\end{array}$ & $\begin{array}{l}\text { Groups evaluated in } \\
\text { different subjects }\end{array}$ & $\mathrm{F} / \mathrm{M}$ & Healthy & 60 & 48 & $\begin{array}{l}\text { Particular } \\
\text { Clinic }\end{array}$ \\
\hline $\begin{array}{l}\text { Chondros et al., } \\
2009\end{array}$ & $\begin{array}{l}\text { Groups evaluated in } \\
\text { different subjects }\end{array}$ & $\mathrm{F} / \mathrm{M}$ & Healthy & 24 & 49 & University \\
\hline $\begin{array}{l}\text { Youssef et al., } \\
2008\end{array}$ & Split mouth & $\mathrm{F} / \mathrm{M}$ & Healthy & 15 & 18,3 & University \\
\hline $\begin{array}{l}\text { Ozcelik et al., } \\
2008\end{array}$ & Split mouth & $\mathrm{F} / \mathrm{M}$ & $\begin{array}{l}\text { Excluded patients with uncontrolled or poorly controlled systemic } \\
\text { conditions }\end{array}$ & 22 & 40 & University \\
\hline $\begin{array}{l}\text { Fernando et al., } \\
1993\end{array}$ & Split mouth & $\mathrm{F} / \mathrm{M}$ & Healthy & 64 & 34 & Hospital \\
\hline
\end{tabular}


Table 2

Qualitative characteristics of the studies related to the treatment, methods and results.

\begin{tabular}{|c|c|c|c|c|c|c|c|c|c|}
\hline Author & $\begin{array}{l}\text { Experimental } \\
\text { model }\end{array}$ & Jaw arch & Region of the jaw & Laser type & $\begin{array}{l}\text { LLLT } \\
\text { parameters }\end{array}$ & $\begin{array}{l}\text { LLLT } \\
\text { application } \\
\text { number } \\
\text { (per } \\
\text { patient) }\end{array}$ & Analysis & $\begin{array}{l}\text { Follow } \\
\text { up } \\
\text { (days) }\end{array}$ & Results \\
\hline $\begin{array}{r}\text { Ferreira et } \\
\text { al., } 2016\end{array}$ & $\begin{array}{l}\text { Maxillary } \\
\text { expansion }\end{array}$ & Maxillar & Anterior & Gaalas & $\begin{array}{l}780 \mathrm{~nm}, \\
35 \mathrm{j} / \mathrm{cm}^{2}, \\
70 \mathrm{mw}, 20 \mathrm{~s}\end{array}$ & 48 & Bone density & 30 & $\begin{array}{l}\text { Positive for all } \\
\text { evaluated } \\
\text { parameters }\end{array}$ \\
\hline $\begin{array}{l}\text { Zaky et al., } \\
2016\end{array}$ & $\begin{array}{l}\text { Maxillary } \\
\text { Cystic } \\
\text { Defects }\end{array}$ & Maxillar & Anterior & Soft laser & $\begin{array}{l}870 \mathrm{~nm}, \\
24 \mathrm{j} / \mathrm{cm}^{2}, \\
50 \mathrm{mw}, 60 \mathrm{~s}\end{array}$ & 7 & Bone density & $\begin{array}{l}1 \text { and } \\
90\end{array}$ & $\begin{array}{l}\text { Positive (at } \\
90 \text { days of } \\
\text { follow up) }\end{array}$ \\
\hline $\begin{array}{l}\text { Abd-Elaal } \\
\text { et al., } \\
2015\end{array}$ & $\begin{array}{l}\text { Distraction } \\
\text { osteogenesis }\end{array}$ & Mandibular & Posterior & Gaas & $\begin{array}{l}905 \mathrm{~nm}, \\
5 \mathrm{j} / \mathrm{cm}^{2}, \\
500 \mathrm{mw}, 120 \mathrm{~s}\end{array}$ & 48 & Bone density & $\begin{array}{l}6,12, \\
24, \\
\text { and } \\
54\end{array}$ & $\begin{array}{l}\text { Positive for all } \\
\text { evaluated } \\
\text { parameters }\end{array}$ \\
\hline $\begin{array}{l}\text { Domínguez } \\
\text { et al., } \\
2015\end{array}$ & $\begin{array}{l}\text { Orthodontic } \\
\text { movement }\end{array}$ & Maxillar & Posterior & Diode & $\begin{array}{l}670 \mathrm{~nm}, 108 \mathrm{j}, \\
200 \mathrm{mw}, 3 \mathrm{~min}\end{array}$ & 18 & $\begin{array}{l}\text { Subjective pain visual scale, tooth } \\
\text { movimentation and bone markers }\end{array}$ & $\begin{array}{l}0,2,4, \\
7,30, \\
\text { and } \\
45\end{array}$ & $\begin{array}{l}\text { No difference } \\
\text { observed }\end{array}$ \\
\hline $\begin{array}{l}\text { Garcia et } \\
\text { al., } 2015\end{array}$ & $\begin{array}{l}\text { Maxillary } \\
\text { expansion }\end{array}$ & Maxillar & Anterior & Ingaalp & $\begin{array}{l}660 \mathrm{~nm}, 26 \\
\text { (ponit a) or } 12 \\
\text { (point b) j/ } \\
\mathrm{cm}^{2}, 100 \mathrm{mw} \text {, } \\
90 \mathrm{~s}\end{array}$ & 56 & $\begin{array}{l}\text { Distance between anterior and } \\
\text { posterior maxillar suture }\end{array}$ & 75 & $\begin{array}{l}\text { Positive for all } \\
\text { evaluated } \\
\text { parameters }\end{array}$ \\
\hline $\begin{array}{l}\text { Eslamian } \\
\text { et al., } \\
2014\end{array}$ & $\begin{array}{l}\text { Orthodontic } \\
\text { movement }\end{array}$ & $\begin{array}{l}\text { Maxillar or } \\
\text { mandibular }\end{array}$ & Posterior & Gaalas & $\begin{array}{l}810 \mathrm{~nm}, \\
2 \mathrm{j} / \mathrm{cm}^{2}, \\
100 \mathrm{mw}, 20 \mathrm{~s}\end{array}$ & 20 & Subjective pain visual scale & $\begin{array}{l}1,3,4, \\
5,6, \\
\text { and } 7\end{array}$ & $\begin{array}{l}\text { Positive for all } \\
\text { evaluated } \\
\text { parameters }\end{array}$ \\
\hline $\begin{array}{r}\text { Mozzati et } \\
\text { al., } 2012\end{array}$ & $\begin{array}{l}\text { Tooth } \\
\text { extraction }\end{array}$ & $\begin{array}{l}\text { Maxillar or } \\
\text { mandibular }\end{array}$ & Anterior/posterior & Gaas & $\begin{array}{l}904-910 \mathrm{~nm}, \\
180 \mathrm{j} / \mathrm{cm}^{2}, \\
200 \mathrm{mw}, \\
15 \mathrm{~min}\end{array}$ & 3 & $\begin{array}{l}\text { Subjective pain visual scale and } \\
\text { biological factors (IL-I } \beta \text {, IL-6, IL-10, } \\
\text { TGF- } \beta 2 \text {, COX-2, BMP- } 4 \text {, BMP-7, } \\
\text { PPAR- } \beta \text {, collagen type I and III) }\end{array}$ & 7 & $\begin{array}{l}\text { Partially } \\
\text { positive (pain } \\
\text { and IL-6, IL-10 } \\
\text { and collagen } \\
\text { type III) }\end{array}$ \\
\hline $\begin{array}{r}\text { Mozzati et } \\
\text { al., } 2011\end{array}$ & $\begin{array}{l}\text { Tooth } \\
\text { extraction }\end{array}$ & $\begin{array}{l}\text { Maxillar or } \\
\text { mandibular }\end{array}$ & Posterior & Gaas & $\begin{array}{l}904-910 \mathrm{~nm}, \\
180 \mathrm{j} / \mathrm{cm}^{2}, \\
200 \mathrm{mw} \\
15 \mathrm{~min}\end{array}$ & 3 & $\begin{array}{l}\text { Subjective pain visual scale and } \\
\text { biological factors (IL-I } \beta \text {, IL-6, IL-10, } \\
\text { TGF- } \beta 2, \text { COX-2, BMP-4, BMP-7, } \\
\text { PPAR- } \beta \text {, collagen type I and III) }\end{array}$ & 7 & $\begin{array}{l}\text { Positive (IL-I } \beta \text {, } \\
\text { IL-6 and COX-2) }\end{array}$ \\
\hline $\begin{array}{l}\text { Angeletti } \\
\text { et al., } \\
2010\end{array}$ & $\begin{array}{l}\text { Maxillary } \\
\text { expansion }\end{array}$ & Maxillar & Anterior & Gaalas & $\begin{array}{l}830 \mathrm{~nm}, \\
140 \mathrm{j} / \mathrm{cm}^{2} \\
100 \mathrm{mw}, 84 \mathrm{~s}\end{array}$ & 24 & Bone density & $\begin{array}{l}30,60 \\
90, \\
120, \\
\text { and } \\
210\end{array}$ & $\begin{array}{l}\text { Positive for all } \\
\text { evaluated } \\
\text { parameters }\end{array}$ \\
\hline $\begin{array}{l}\text { AboElsaad } \\
\text { et al., } \\
2009\end{array}$ & $\begin{array}{l}\text { Periodontal } \\
\text { defects }\end{array}$ & $\begin{array}{l}\text { Maxillar or } \\
\text { mandibular }\end{array}$ & Posterior & Gaalas & $\begin{array}{l}830 \mathrm{~nm}, \\
4 \mathrm{j} / \mathrm{cm}^{2}, \\
40 \mathrm{mw}, 60 \mathrm{~s}\end{array}$ & 4 & $\begin{array}{l}\text { Bone area and clinical parameters } \\
\text { (probing depth and attachment } \\
\text { level) }\end{array}$ & $\begin{array}{l}90 \\
\text { and } \\
180\end{array}$ & $\begin{array}{l}\text { Partially } \\
\text { positive (at } \\
90 \text { days of } \\
\text { follow up) }\end{array}$ \\
\hline $\begin{array}{l}\text { Angelov et } \\
\text { al., } 2009\end{array}$ & $\begin{array}{l}\text { Periodontal } \\
\text { defects }\end{array}$ & $\begin{array}{l}\text { Maxillar } \\
\text { and } \\
\text { mandibular }\end{array}$ & Anterior/posterior & Diode & $\begin{array}{l}630-670 \mathrm{~nm}, \\
1.875 \mathrm{j} / \mathrm{cm}^{2} \\
25 \mathrm{mw}, 16 \mathrm{~min}\end{array}$ & 10 & $\begin{array}{l}\text { Clinical parameters (plaque, } \\
\text { gingival and sulcular bleeding } \\
\text { indexes) }\end{array}$ & 30 & $\begin{array}{l}\text { Positive for all } \\
\text { evaluated } \\
\text { parameters }\end{array}$ \\
\hline $\begin{array}{l}\text { Chondros } \\
\text { et al., } \\
2009\end{array}$ & $\begin{array}{l}\text { Periodontal } \\
\text { defects }\end{array}$ & $\begin{array}{l}\text { Maxillar } \\
\text { and } \\
\text { mandibular }\end{array}$ & Anterior/posterior & Diode & $\begin{array}{l}670 \mathrm{~nm}, \\
75 \mathrm{mw} / \mathrm{cm}^{2} \\
60 \mathrm{~s}\end{array}$ & 1 & $\begin{array}{l}\text { Clinical parameters (probing depth, } \\
\text { Recession of the gingival margin, } \\
\text { attachment level, plaque and } \\
\text { bleeding scores) }\end{array}$ & $\begin{array}{l}90 \\
\text { and } \\
180\end{array}$ & $\begin{array}{l}\text { No difference } \\
\text { observed }\end{array}$ \\
\hline $\begin{array}{r}\text { Youssef et } \\
\text { al., } 2008\end{array}$ & $\begin{array}{l}\text { Orthodontic } \\
\text { movement }\end{array}$ & $\begin{array}{l}\text { Maxillar } \\
\text { and } \\
\text { mandibular }\end{array}$ & Posterior & Gaalas & $\begin{array}{l}809 \mathrm{~nm}, \\
8 \mathrm{j} / \mathrm{cm}^{2} \\
100 \mathrm{mw}, 40 \mathrm{~s}\end{array}$ & 4 & $\begin{array}{l}\text { Subjective pain visual scale and } \\
\text { tooth movimentation }\end{array}$ & 60 & $\begin{array}{l}\text { Positive for all } \\
\text { evaluated } \\
\text { parameters }\end{array}$ \\
\hline $\begin{array}{l}\text { Ozcelik et } \\
\text { al., } 2008\end{array}$ & $\begin{array}{l}\text { Periodontal } \\
\text { defects }\end{array}$ & Mandibular & Posterior & Diode & $\begin{array}{l}588 \mathrm{~nm}, \\
4 \mathrm{j} / \mathrm{cm}^{2}, 10 \mathrm{~min}\end{array}$ & 6 & $\begin{array}{l}\text { Subjective pain visual scale, clinical } \\
\text { parameters (swelling, collour, } \\
\text { bleeding, gingival recession, } \\
\text { probing depth and attachment } \\
\text { level) }\end{array}$ & $\begin{array}{l}30,60 \\
180 \\
\text { and } \\
360\end{array}$ & $\begin{array}{l}\text { Partially } \\
\text { positive } \\
\text { (gingival } \\
\text { recession, } \\
\text { swelling and } \\
\text { pain) }\end{array}$ \\
\hline $\begin{array}{l}\text { Fernando } \\
\text { et al., } \\
1993\end{array}$ & $\begin{array}{l}\text { Tooth } \\
\text { extraction }\end{array}$ & Mandibular & Posterior & Semi-conductor & $\begin{array}{l}830 \mathrm{~nm}, \\
4 \mathrm{j} / \mathrm{cm}^{2}, \\
30 \mathrm{mw}, 132 \mathrm{~s}\end{array}$ & 1 & $\begin{array}{l}\text { Postoperative pain scale, swelling } \\
\text { and healing }\end{array}$ & $\begin{array}{l}1,3 \\
\text { and } 7\end{array}$ & $\begin{array}{l}\text { No difference } \\
\text { observed }\end{array}$ \\
\hline
\end{tabular}

\section{Results}

\subsection{Literature Search}

The database search retrieved 518 references, including 293 from PubMed/Medline, 210 from Scopus, and 14 from the Cochrane Library. After applying the inclusion/exclusion criteria to the titles and abstracts of the selected comparative studies, 17 studies remained. Reading these study texts resulted in exclusion of 8 more studies because they were performed in less than 10 patients [22], had an unclear description of/ or unappropriate randomization [39,40,5,7], had subjective criteria for evaluating results [13], maxillary expansion with follow-up less than 30 days [10] and was a prospective study [41]. A manual search for articles identified six more studies. Overall, 15 studies [1,6,8,9,14,15,42-50] were selected for the analysis (Tables 1 and 2). Details of the search strategy are presented in Fig. 1.

The kappa inter-investigator agreement for articles that were selected from PubMed/MEDLINE (kappa value $=0.71$ ), Scopus (kappa 
Table 3

Qualitative characteristics of the studies that quantitatively evaluated parameters related to bone healing.

\begin{tabular}{|c|c|c|c|c|c|c|c|c|c|c|}
\hline Author & $\begin{array}{l}\text { Experimental } \\
\text { model }\end{array}$ & Jaw arch & $\begin{array}{l}\text { Region } \\
\text { of the } \\
\text { jaw }\end{array}$ & $\begin{array}{l}\text { Laser } \\
\text { type }\end{array}$ & LLLT parameters & $\begin{array}{l}\text { LLLT application } \\
\text { number (per } \\
\text { patient) }\end{array}$ & analysis & Parameter evaluated & $\begin{array}{l}\text { Follow up } \\
\text { (days) }\end{array}$ & Results \\
\hline $\begin{array}{l}\text { Ferreira et } \\
\text { al., } \\
2016\end{array}$ & $\begin{array}{l}\text { Maxillary } \\
\text { expansion }\end{array}$ & Maxillar & Anterior & Gaalas & $\begin{array}{l}780 \mathrm{~nm}, 35 \mathrm{~J} / \mathrm{cm}^{2}, \\
70 \mathrm{~mW}, 20 \mathrm{~s}\end{array}$ & 48 & Tomography & Bone density & 30 & $\begin{array}{l}\text { Positive for all } \\
\text { evaluated } \\
\text { parameters }\end{array}$ \\
\hline $\begin{array}{l}\text { Zaky et } \\
\text { al., } \\
2016\end{array}$ & $\begin{array}{l}\text { Maxillary } \\
\text { cystic defects }\end{array}$ & Maxillar & Anterio & $\begin{array}{l}\text { Soft } \\
\text { laser }\end{array}$ & $\begin{array}{l}870 \mathrm{~nm}, 24 \mathrm{~J} / \mathrm{cm}^{2}, \\
50 \mathrm{~mW}, 60 \mathrm{~s}\end{array}$ & 7 & Radiograph & Bone density & 1 and 90 & $\begin{array}{l}\text { Positive (at } \\
90 \text { days of } \\
\text { follow up) }\end{array}$ \\
\hline $\begin{array}{l}\text { Abd-Elaal } \\
\text { et al., } \\
2015\end{array}$ & $\begin{array}{l}\text { Distraction } \\
\text { osteogenesis }\end{array}$ & Mandibular & Posterior & Gaas & $\begin{array}{l}905 \mathrm{~nm}, 5 \mathrm{~J} / \mathrm{cm}^{2} \text {, } \\
500 \mathrm{~mW}, 120 \mathrm{~s}\end{array}$ & 48 & Radiograph & Bone density & $\begin{array}{l}6,12,24, \\
\text { and } 54\end{array}$ & $\begin{array}{l}\text { Positive for all } \\
\text { evaluated } \\
\text { parameters }\end{array}$ \\
\hline $\begin{array}{l}\text { Garcia et } \\
\text { al. } 2015\end{array}$ & $\begin{array}{l}\text { Maxillary } \\
\text { expansion }\end{array}$ & Maxillar & Anterior & Ingaalp & $\begin{array}{l}660 \mathrm{~nm}, 26 \text { (ponit A) or } \\
12 \text { (point B) J/cm², } \\
100 \mathrm{~mW}, 90 \mathrm{~s}\end{array}$ & 56 & Tomography & $\begin{array}{l}\text { Distance between } \\
\text { anterior and } \\
\text { posterior suture }\end{array}$ & 75 & $\begin{array}{l}\text { Positive for all } \\
\text { evaluated } \\
\text { parameters }\end{array}$ \\
\hline $\begin{array}{l}\text { Angeletti } \\
\text { et al., } \\
2010\end{array}$ & $\begin{array}{l}\text { Maxillary } \\
\text { expansion }\end{array}$ & Maxillar & Anterior & Gaalas & $\begin{array}{l}830 \mathrm{~nm}, 140 \mathrm{~J} / \mathrm{cm}^{2} \\
100 \mathrm{~mW}, 84 \mathrm{~s}\end{array}$ & 24 & Radiograph & Bone density & $\begin{array}{l}30,60,90 \\
120, \text { and } \\
210\end{array}$ & $\begin{array}{l}\text { Positive for all } \\
\text { evaluated } \\
\text { parameters }\end{array}$ \\
\hline $\begin{array}{l}\text { AboElsaad } \\
\text { et al., } \\
2009\end{array}$ & $\begin{array}{l}\text { Periodontal } \\
\text { defects }\end{array}$ & $\begin{array}{l}\text { Maxillar } \\
\text { and } \\
\text { mandibullar }\end{array}$ & Posterior & Gaalas & $\begin{array}{l}830 \mathrm{~nm}, 4 \mathrm{~J} / \mathrm{cm}^{2}, 40 \mathrm{~mW} \text {, } \\
60 \mathrm{~s}\end{array}$ & 4 & Radiograph & Bone area & $\begin{array}{l}90 \text { and } \\
180\end{array}$ & $\begin{array}{l}\text { Positive (at } \\
90 \text { days of } \\
\text { follow up) }\end{array}$ \\
\hline
\end{tabular}

value $=0.70$ ), and the Cochrane Library (kappa value $=1.00$ ) showed an acceptable level of agreement [51].

\subsection{Characteristics of the Included Studies Related to Patients}

A total of 374 patients were treated with LLLT, and they had a mean age of 28.5 years. Four studies were conducted in hospitals $[8,14,42,44]$, one study was conducted in a clinic [47], 9 studies were conducted at universities and one study was conducted in a researcher center [50]. All studies included patients of both sexes. Eight studies applied the "split mouth" model as an experimental design [6,8,15,42,43,45,46,49] and in the others, researchers evaluated experimental groups in different subjects. Most of the studies included that they enrolled healthy patients with no systemic conditions. Only three studies did not fall under these conditions: Ozcelik et al. [15] excluded patients with uncontrolled or poorly controlled diabetes, pregnancy, or any other systemic diseases known to affect periodontal tissues; Mozzati et al. [46] selected subjects waiting for liver transplantation; Zaky et al., (2016) excluded subjects if they have any systemic disease that interferes with bone healing [50]. Assessment of the included studies are described in Fig. 2.

\subsection{Characteristics of the Included Studies Related to Treatment, Methods, and Results}

The more commonly used laser device was gallium aluminium arsenide (GaAlAs), which was used in five studies [1,9,14,45,49], followed by (gallium arsenide (GaAs) [6,42,46]. Other types of lasers described included diode [15,43], aluminium-gallium indium-phosphide (InGaAlP) [44], semi-conductor [8] and soft laser [50].

The wavelengths used also varied greatly. Studies used lasers with a wavelength of $500 \mathrm{~nm}$ [15], $600 \mathrm{~nm}[43,44,47,48], 700 \mathrm{~nm}$ [9], $800 \mathrm{~nm}$ $[1,8,14,45,49,50]$, or $900 \mathrm{~nm}[6,42,46]$.

Table 4

Quality assessment of the selected studies by JADAD scale.

\begin{tabular}{|c|c|c|c|c|c|c|c|c|c|c|c|c|c|c|c|}
\hline \multirow[b]{2}{*}{ Quality criteria } & \multicolumn{15}{|l|}{ Studies } \\
\hline & $\begin{array}{l}\text { Ferreira } \\
\text { et al., } \\
2016\end{array}$ & $\begin{array}{l}\text { Zaky } \\
\text { et } \\
\text { al., } \\
2016\end{array}$ & $\begin{array}{l}\text { Abd-Elaal } \\
\text { et al., } \\
2015\end{array}$ & $\begin{array}{l}\text { Domínguez } \\
\text { et al., } 2015\end{array}$ & $\begin{array}{l}\text { Garcia } \\
\text { et al., } \\
2015\end{array}$ & $\begin{array}{l}\text { Eslamian } \\
\text { et al., } \\
2014\end{array}$ & $\begin{array}{l}\text { Mozzati } \\
\text { et al., } \\
2012\end{array}$ & $\begin{array}{l}\text { Angelov } \\
\text { et al., } \\
2009\end{array}$ & $\begin{array}{l}\text { Mozzati } \\
\text { et al., } \\
2011\end{array}$ & $\begin{array}{l}\text { Angeletti } \\
\text { et al., } \\
2010\end{array}$ & $\begin{array}{l}\text { AboElsaad } \\
\text { et al., } \\
2009\end{array}$ & $\begin{array}{l}\text { Chondros } \\
\text { et al., } \\
2009\end{array}$ & $\begin{array}{l}\text { Youssef } \\
\text { et al., } \\
2008\end{array}$ & $\begin{array}{l}\text { Ozcelik } \\
\text { et al., } \\
2008\end{array}$ & $\begin{array}{l}\text { Fernando } \\
\& \\
\text { Walker, } \\
1993\end{array}$ \\
\hline $\begin{array}{l}\text { 1. Was the study } \\
\text { described as } \\
\text { random? }\end{array}$ & $\mathrm{Y}$ & $\mathrm{Y}$ & Y & $\mathrm{Y}$ & Y & $\mathrm{Y}$ & $\mathrm{Y}$ & $\mathrm{Y}$ & Y & $\mathrm{Y}$ & $\mathrm{Y}$ & Y & Y & $\mathrm{Y}$ & Y \\
\hline $\begin{array}{l}\text { 2. Was the } \\
\text { randomization } \\
\text { scheme } \\
\text { described and } \\
\text { appropriate? }\end{array}$ & $\mathrm{Y}$ & Y & $\mathrm{Y}$ & $\mathrm{Y}$ & $\mathrm{Y}$ & $\mathrm{Y}$ & Y & Y & Y & $\mathrm{N}$ & $\mathrm{Y}$ & Y & $\mathrm{Y}$ & $\mathrm{Y}$ & Y \\
\hline $\begin{array}{l}\text { 3. Was the study } \\
\text { described as } \\
\text { double-blind? }\end{array}$ & $\mathrm{N}$ & $\mathrm{N}$ & $\mathrm{N}$ & $\mathrm{N}$ & $\mathrm{N}$ & $\mathrm{N}$ & $\mathrm{N}$ & $\mathrm{N}$ & $\mathrm{N}$ & $\mathrm{N}$ & $\mathrm{N}$ & $\mathrm{N}$ & $\mathrm{N}$ & $\mathrm{Y}$ & $\mathrm{Y}$ \\
\hline $\begin{array}{l}\text { 4. Was the } \\
\text { method of } \\
\text { double } \\
\text { blinding } \\
\text { appropriate? }\end{array}$ & $\mathrm{N}$ & $\mathrm{N}$ & $\mathrm{N}$ & $\mathrm{N}$ & $\mathrm{N}$ & $\mathrm{N}$ & $\mathrm{N}$ & $\mathrm{N}$ & $\mathrm{N}$ & $\mathrm{N}$ & $\mathrm{N}$ & $\mathrm{N}$ & $\mathrm{N}$ & $\mathrm{N}$ & Y \\
\hline $\begin{array}{l}\text { 5. Was there a } \\
\text { description of } \\
\text { dropouts and } \\
\text { withdrawals? }\end{array}$ & $\mathrm{Y}$ & Y & $\mathrm{Y}$ & $\mathrm{Y}$ & Y & $\mathrm{Y}$ & $\mathrm{Y}$ & Y & $\mathrm{Y}$ & $\mathrm{Y}$ & $\mathrm{Y}$ & Y & $\mathrm{Y}$ & $\mathrm{Y}$ & Y \\
\hline Jadad score & 3 & 3 & 3 & 3 & 3 & 3 & 3 & 3 & 3 & 2 & 3 & 3 & 3 & 4 & 5 \\
\hline Quality of study & High & Hihg & High & High & High & High & High & High & High & Low & High & High & High & High & High \\
\hline
\end{tabular}

Abrevitations: Y, Yes; N, No. 


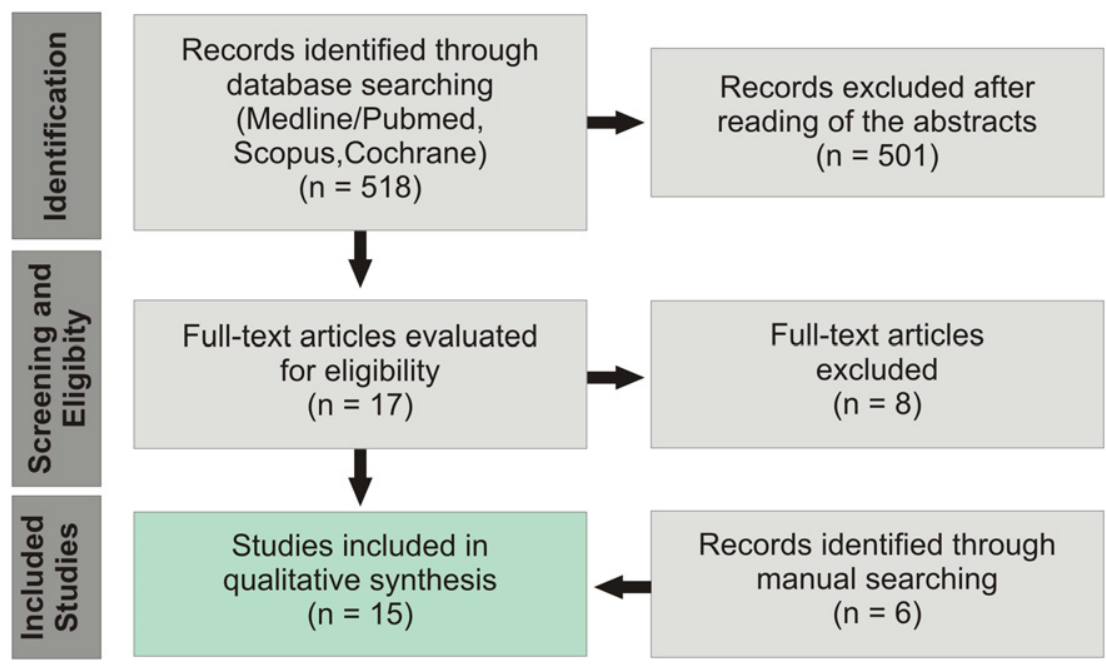

Fig. 1. Literature search diagram flow.

Follow-up varied from 0 to 360 days. The total number of LLLT applications was 251. Maxillar arch was more frequently evaluated among the studies and LLLT applications were performed more frequently in the posterior regions of the jaw. The experimental models evaluated were tooth extraction, distraction osteogenesis, maxillary expansion, periodontal defects, orthodontic movement and maxillary cystic defects.

From 15 selected studies, six evaluated parameters of the results specifically related to bone healing that is the subject of the present systematic review $[1,9,14,42,44,50]$ (Table 3 ). Of these, four studies showed improvement directly related to bone formation (primary outcomes) in the groups treated with LLLT for all evaluated parameters [1, $9,42,44]$ and two studies demonstrated improved results in one of the follow up periods $[14,50]$. The other nine studies $[6,8,15,43,45,46,47$, 48,49 ] evaluated secondary parameters related to healing (secondary outcomes) in the maxillofacial area after application of LLLT, such as biological markers, anti-inflammatory effect, analgesia, and tissue healing, in addition to a quality of life questionnaire related to oral health. There were no reports of adverse or negative effects of LLLT.

\section{Discussion}

LLLT has been reported to be an effective tool for the treatment of postsurgical conditions because of its analgesic and anti-inflammatory effects, and because of its stimulating effect on tissue healing [5]. In recent years, there has been much research on LLLT for the Dentistry field [26], but the benefits of LLLT are controversial. Thus, we systematically investigated the results published on the effect of this therapeutic approach on bone repair in the field of Dentistry.

The present review included only studies that clinically evaluated bone defects in the maxillofacial area that were treated with lasers and compared to a control group. The quantitative analyses varied greatly in the included studies. Although LLLT was used to improve bone defect healing in the maxillofacial area, only six of the 15 selected

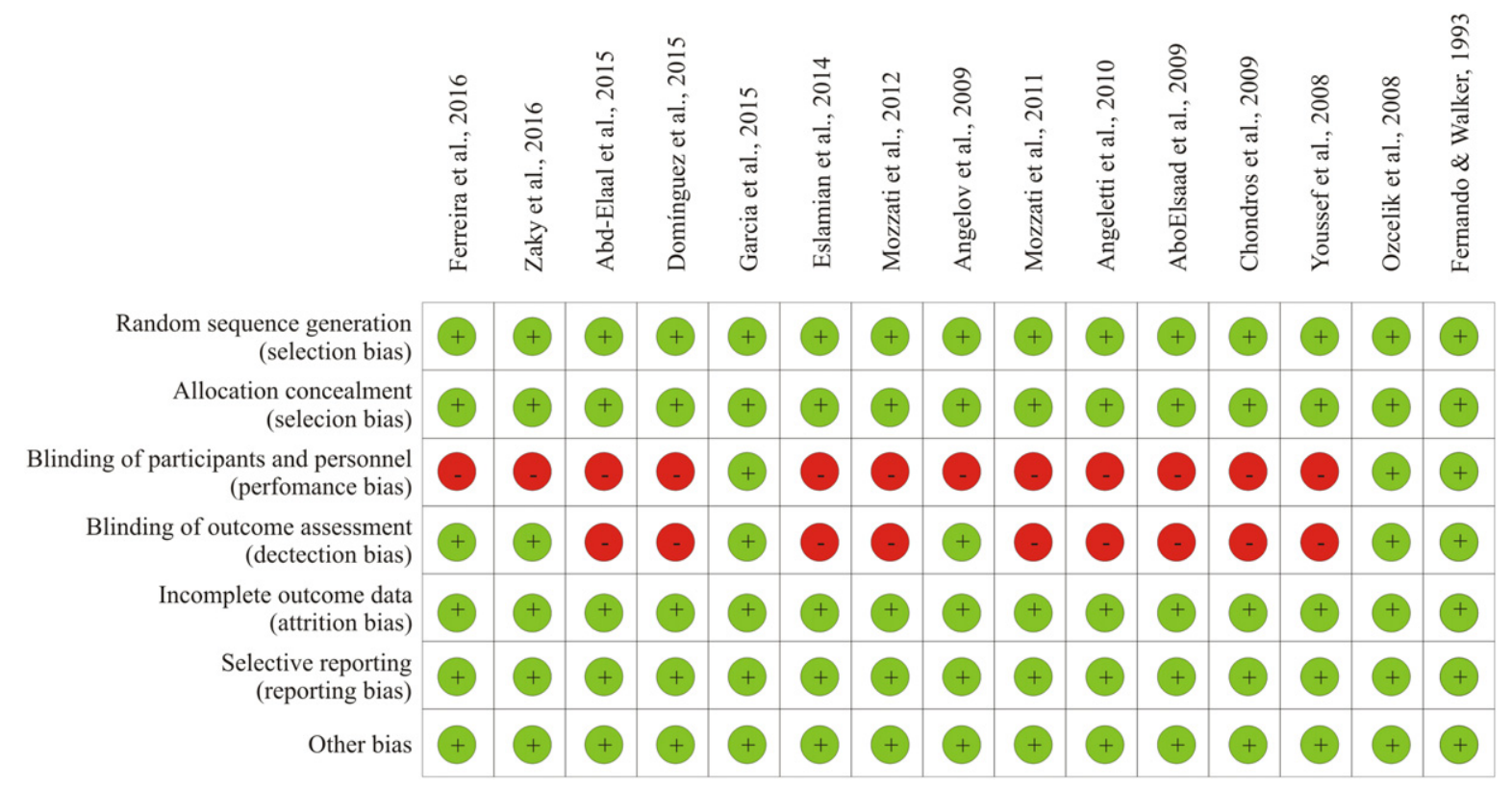

Fig. 2. Assessing risk of bias in included studies by Cochrane risk of bias tools. 
studies evaluated primary outcomes (bone formation). Of these, four studies evaluated bone formation using radiographs $[1,14,42,50]$ and two studies assessed bone formation topographically $[9,44]$ (Table 3 ). Measurement units were used and data were presented in different ways by the authors. Therefore, a quantitative analysis (meta-analysis) of the present systematic review results could not be performed.

In the qualitative analysis, most of the included studies presented positive primary or secondary outcomes with LLLT treatment in the maxillofacial area. Of these studies, three reported experimental groups treated with LLLT with any improvement in the results $[8,43,48]$. It is important to consider that application frequency and specific combinations of LLLT parameters can influence the results observed. This suggestion can be confirmed by an in vitro study that demonstrates that various osteogenic cell strains respond differently to specific combinations of wavelengths and doses [52]. Additionally, studies where several applications of LLLT were used showed positive results [9,42,44]. However, although the LLLT protocol used in these studies did not promote bone regeneration, there were also no negative effects.

The importance of choosing an adequate energy level has been discussed by several authors [17,53,54], but an ideal protocol for LLLT use has not yet been obtained for promoting bone formation. This can be attributed to different laser types that are available and the different application protocols that are used as a result. However, it is important to consider that positive outcomes have been obtained. Most of the studies included in the present systematic review reported a primary and/or secondary outcome that showed that LLLT treatment promotes an improvement in bone healing in the maxillofacial area $[1,9,14,42$, $44,50]$. Thus, the null hypothesis that no differences are found between bone defects treated with lasers compared to a control group was rejected.

In this systematic review, various experimental models, in addition to an RCT design, were selected. Thus, age, absence/presence of local infection, follow up, bleeding, saliva, and jaw arch involved in the treatment influenced the observed results. Age was a factor that varied greatly, because it was evaluated the effect of LLLT after maxillary expansion in patients with a mean age of 10.5 years [9], while others studies assessed periodontal defects or tooth extraction in patients with a mean age of 42.2 years $[14,15]$. Additionally, the studies that evaluated primary outcomes and used follow up periods compatible with the time needed for bone formation reported positive results $[1,9,42]$. Other studies, however, did not observe these benefits. Abo-Elsaad et al. [14] assessed healing of periodontal defects with bioactive glass combined or not with LLLT and evaluated the results through radiographs at 90 and 180 days postoperative. The authors observed improved results only at 90 days postoperatively. Radiographic evaluation of tooth extraction sockets with or without LLLT treatment at baseline and at postoperative day 180 has suggested no benefits with the use of LLLT [7]. Perhaps if this evaluation assessed a time point in the middle, it would have found that LLLT accelerated bone healing, even when there was no statistical difference between the groups at the end of the study period [7]. In this context, is important to highlight the secondary outcomes, which also had positive results after LLLT treatment in maxillofacial area conditions. The anti-inflammatory, analgesic and healing acceleration effects, as well as quality of life related to oral health in patients who underwent LLLT suggest that this is a promising therapy that is easy to use, and has a relatively low cost, and which favors a reduction in the postoperative administration of medicine for dental patients. This therapy can show a benefit in secondary outcomes in a large portion of the population whether patients have systemic involvement, or if they are elderly people for whom additional care is required to administer medicine.

Finally, some studies did not describe all the LLLT parameters and important data of the subjects in the methodology section $[15,46,48$, 50]. A wide variation in the energy density, number of applications, wavelength used, power, and other LLLT specifications has been reported. This information is of important for readers and researchers to be able to evaluate the studies' results and the specific treatment applied. The results should be interpreted with caution because of uncontrolled factors in the included studies. Therefore, additional RCTs should be performed confirm the effectiveness of LLLT in improving bone density in human maxillofacial defects.

\section{Conclusion}

Within the limitation of this review, a possible improvement in bone density can be found when LLLT is applied postoperatively in maxillofacial bony defects. LLLT also seems to promote anti-inflammatory and analgesic effects and accelerate healing, as well as enhance quality of life related to oral health. However, LLLT use protocols need to be standardized before more specific conclusions can be drawn about this subject.

\section{References}

[1] P. Angeletti, M.D. Pereira, H.C. Gomes, C.T. Hino, L.M. Ferreira, Effect of low-leve laser therapy (GaAlAs) on bone regeneration in midpalatal anterior suture after surgically assisted rapid maxillary expansion, Oral Surg. Oral Med. Oral Pathol. Oral Radiol. Endod. 109 (3) (2010 Mar) e38-e46 (PubMed PMID: 20219584. Epub 2010/03/12. eng)

[2] T.C. Keaney, E. Tanzi, T. Alster, Comparison of $532 \mathrm{~nm}$ potassium titanyl phosphate laser and $595 \mathrm{~nm}$ pulsed dye laser in the treatment of erythematous surgical scars: a randomized, controlled, Open-label study, Dermatol. Surg. 42 (1) (2016 Jan) 70-76 (PubMed PMID: 26673432. Epub 2015/12/18. eng)s.

[3] J.A. Gladsjo, S.I. Jiang, Treatment of surgical scars using a 595-nm pulsed dye laser using purpuric and nonpurpuric parameters: a comparative study, Dermatol. Surg 40 (2) (2014 Feb) 118-126 (PubMed PMID: 24373006. Epub 2014/01/01. eng).

[4] W.D. Chang, J.H. Wu, H.J. Wang, J.A. Jiang, Therapeutic outcomes of low-level laser therapy for closed bone fracture in the human wrist and hand, Photomed. Laser Surg. 32 (4) (2014 Apr) 212-218 (PubMed PMID: 24649935. Epub 2014/03/22. eng).

[5] G. Batinjan, Z. Zore, A. Celebic, M. Papic, D. Gabric Panduric, Zore I. Filipovic, Thermographic monitoring of wound healing and oral health-related quality of life in patients treated with laser (aPDT) after impacted mandibular third molar removal, Int. J. Oral Maxillofac. Surg. 43 (12) (2014 Dec) 1503-1508 (PubMed PMID: 25277807. Epub 2014/10/04. eng).

[6] M. Mozzati, G. Martinasso, N. Cocero, R. Pol, M. Maggiora, G. Muzio, et al., Influence of superpulsed laser therapy on healing processes following tooth extraction, Photomed. Laser Surg. 29 (8) (2011 Aug) 565-571 (PubMed PMID: 21631375. Epub 2011/06/03. Eng).

[7] H. Kucerova, T. Dostalova, L. Himmlova, J. Bartova, J. Mazanek, Low-level laser therapy after molar extraction, J. Clin. Laser Med. Surg. 18 (6) (2000 Dec) 309-315 (PubMed PMID: 11572225. Epub 2001/09/27. eng).

[8] S. Fernando, C.M. Hill, R. Walker, A randomised double blind comparative study of low level laser therapy following surgical extraction of lower third molar teeth, Br. J. Oral Maxillofac. Surg. 31 (3) (1993 Jun) 170-172 (PubMed PMID: 8512911. Epub 1993/06/01. eng).

[9] F.N. Ferreira, J.O. Gondim, J.J. Neto, P.C. Dos Santos, K.M. de Freitas Pontes, L.M. Kurita, et al., Effects of low-level laser therapy on bone regeneration of the midpalatal suture after rapid maxillary expansion, Lasers Med. Sci. 31 (5) (2016 Jul) 907-913 (PubMed PMID: 27056702. Epub 2016/04/09. eng).

[10] F. Cepera, F.C. Torres, M.A. Scanavini, L.R. Paranhos, L. Capelozza Filho, M.A. Cardoso, et al., Effect of a low-level laser on bone regeneration after rapid maxillary expansion, Am. J. Orthod. Dentofac. Orthop. 141 (4) (2012 Apr) 444-450 (PubMed PMID: 22464526. Epub 2012/04/03. eng).

[11] S.B. Dias, M.V. Fonseca, N.C. Dos Santos, I.F. Mathias, F.C. Martinho, M.S. Junior, et al., Effect of GaAIAs low-level laser therapy on the healing of human palate mucosa after connective tissue graft harvesting: randomized clinical trial, Lasers Med. Sci. 30 (6) (2015 Aug) 1695-1702 (PubMed PMID: 25373688. Epub 2014/11/07. eng).

[12] P. Vescovi, M. Manfredi, E. Merigo, M. Meleti, C. Fornaini, J.P. Rocca, et al., Surgical approach with Er:YAG laser on osteonecrosis of the jaws (ONJ) in patients under bisphosphonate therapy (BPT), Lasers Med. Sci. 25 (1) (2010 Jan) 101-113 (PubMed PMID: 19543768. Epub 2009/06/23. eng).

[13] B. Atalay, S. Yalcin, Y. Emes, I. Aktas, B. Aybar, H. Issever, et al., Bisphosphonate-related osteonecrosis: laser-assisted surgical treatment or conventional surgery? Lasers Med. Sci. 26 (6) (2011 Nov) 815-823 (PubMed PMID: 21809068. Epub 2011/08/ 03. eng).

[14] N.S. AboElsaad, M. Soory, L.M. Gadalla, L.I. Ragab, S. Dunne, K.R. Zalata, et al., Effect of soft laser and bioactive glass on bone regeneration in the treatment of infra-bony defects (a clinical study), Lasers Med. Sci. 24 (3) (2009 May) 387-395 (PubMed PMID: 18581160. Epub 2008/06/27. eng).

[15] O. Ozcelik, M. Cenk Haytac, G. Seydaoglu, Enamel matrix derivative and low-leve laser therapy in the treatment of intra-bony defects: a randomized placebo-controlled clinical trial, J. Clin. Periodontol. 35 (2) (2008 Feb) 147-156 (PubMed PMID: 18081859. Epub 2007/12/18. eng).

[16] G.G. Walmsley, R.C. Ransom, E.R. Zielins, T. Leavitt, J.S. Flacco, M.S. Hu, et al., Stem cells in bone regeneration, Stem Cell Rev. (2016 Jun 1) (PubMed PMID: 27250635. Epub 2016/06/03. Eng). 
17] M.J. Nagata, C.S. Santinoni, N.M. Pola, N. de Campos, M.R. Messora, S.R. Bomfim, et al., Bone marrow aspirate combined with low-level laser therapy: a new therapeutic approach to enhance bone healing, J. Photochem. Photobiol. B 121 (2013 Apr 5) 6-14 (PubMed PMID: 23474527. Epub 2013/03/12. eng).

[18] M. Khadra, S.P. Lyngstadaas, H.R. Haanaes, K. Mustafa, Effect of laser therapy on attachment, proliferation and differentiation of human osteoblast-like cells cultured on titanium implant material, Biomaterials 26 (17) (2005 Jun) 3503-3509 (PubMed PMID: 15621240. Epub 2004/12/29. eng).

[19] J.B. Park, S.J. Ahn, Y.G. Kang, E.C. Kim, J.S. Heo, K.L. Kang, Effects of increased lowlevel diode laser irradiation time on extraction socket healing in rats, Lasers Med. Sci. (2013 Aug 9) (PubMed PMID: 23929563. Epub 2013/08/10. Eng).

[20] P.S. Bossini, A.C. Renno, D.A. Ribeiro, R. Fangel, A.C. Ribeiro, A. Lahoz Mde, et al., Low level laser therapy $(830 \mathrm{~nm})$ improves bone repair in osteoporotic rats: similar outcomes at two different dosages, Exp. Gerontol. 47 (2) (2012 Feb) 136-142 (PubMed PMID: 22138375. Epub 2011/12/06. eng).

[21] N.A. Fujihara, K.R. Hiraki, M.M. Marques, Irradiation at $780 \mathrm{~nm}$ increases proliferation rate of osteoblasts independently of dexamethasone presence, Lasers Surg. Med. 38 (4) (2006 Apr) 332-336 (PubMed PMID: 16526043. Epub 2006/03/10. eng).

[22] J.M. Garcia-Morales, P. Tortamano-Neto, F.F. Todescan, J.C. de Andrade, J. Marotti Jr. D.M. Zezell, Stability of dental implants after irradiation with an 830-nm low-level laser: a double-blind randomized clinical study, Lasers Med. Sci. 27 (4) (2012 Jul) 703-711 (PubMed PMID: 21732113. Epub 2011/07/07. eng).

[23] N. Jakse, M. Payer, S. Tangl, A. Berghold, R. Kirmeier, M. Lorenzoni, Influence of lowlevel laser treatment on bone regeneration and osseointegration of dental implants following sinus augmentation. An experimental study on sheep, Clin. Oral Implants Res. 18 (4) (2007 Aug) 517-524 (PubMed PMID: 17451409. Epub 2007/04/25. eng).

[24] S. Bashardoust Tajali, J.C. Macdermid, P. Houghton, R. Grewal, Effects of low power laser irradiation on bone healing in animals: a meta-analysis, J. Orthop. Surg. Res. 5 (2010) 1 (PubMed PMID: 20047683. PMCID: Pmc2829511. Epub 2010/01/06. eng.

[25] R. Amid, M. Kadkhodazadeh, M.G. Ahsaie, A. Hakakzadeh, Effect of low level laser therapy on proliferation and differentiation of the cells contributing in bone regeneration, J. Lasers Med. Sci. 5 (4) (2014) 163-170 (PubMed PMID: 25653816. PMCID: PMC4281990. eng)

[26] J.C. Prados-Frutos, J. Rodriguez-Molinero, M. Prados-Privado, J.H. Torres, R. Rojo, Lack of clinical evidence on low-level laser therapy (LLLT) on dental titanium implant: a systematic review, Lasers Med. Sci. 31 (2) (2016 Feb) 383-392 (PubMed PMID: 26754180. Epub 2016/01/13. eng).

[27] Z.S. Natto, M. Aladmawy, P.A. Levi Jr., H.L. Wang, Comparison of the efficacy of different types of lasers for the treatment of peri-implantitis: a systematic review, Int. J. Oral Maxillofac. Implants 30 (2) (2015 Mar-Apr) 338-345 (PubMed PMID: 25830394. Epub 2015/04/02. eng).

[28] C. Ren, C. McGrath, L. Jin, C. Zhang, Y. Yang, The effectiveness of low-level laser therapy as an adjunct to non-surgical periodontal treatment: a meta-analysis, J. Periodontal Res. 2 (2016 Mar) (PubMed PMID: 26932392. Epub 2016/03/05. Eng)

[29] D.E. Slot, K.H. Jorritsma, C.M. Cobb, F.A. Van der Weijden, The effect of the therma diode laser (wavelength $808-980 \mathrm{~nm}$ ) in non-surgical periodontal therapy: a systematic review and meta-analysis, J. Clin. Periodontol. 41 (7) (2014 Jul) 681-692 (PubMed PMID: 24460795. Epub 2014/01/28. eng)

[30] F. Sgolastra, M. Severino, R. Gatto, A. Monaco, Effectiveness of diode laser as adjunctive therapy to scaling root planning in the treatment of chronic periodontitis: a meta-analysis, Lasers Med. Sci. 28 (5) (2013 Sep) 1393-1402 (PubMed PMID: 22895576. Epub 2012/08/17. eng).

[31] Z. Kalemaj, I.C. Debernard, J. Buti, Efficacy of surgical and non-surgical interventions on accelerating orthodontic tooth movement: a systematic review, Eur J Oral Implantol. 8 (1) (2015 Spring) 9-24 (PubMed PMID: 25738176. Epub 2015/03/05 eng).

[32] M.K. Ge, W.L. He, J. Chen, C. Wen, X. Yin, Z.A. Hu, et al., Efficacy of low-level laser therapy for accelerating tooth movement during orthodontic treatment: a systematic review and meta-analysis, Lasers Med. Sci. 30 (5) (2015 Jul) 1609-1618 (PubMed PMID: 24554452. Epub 2014/02/21. eng)

[33] A.A. Khan, A. Morrison, D.A. Hanley, D. Felsenberg, L.K. McCauley, F. O'Ryan, et al., Diagnosis and management of osteonecrosis of the jaw: a systematic review and international consensus, J. Bone Miner. Res. 30 (1) (2015 Jan) 3-23 (PubMed PMID: 25414052. Epub 2014/11/22. eng).

[34] C. Doeuk, B. Hersant, R. Bosc, F. Lange, M. SidAhmed-Mezi, J. Bouhassira, et al., Current indications for low level laser treatment in maxillofacial surgery: a review, $\mathrm{Br}$. . Oral Maxillofac. Surg. 53 (4) (2015 Apr) 309-315 (PubMed PMID: 25740083. Epub 2015/03/06. eng)

[35] D. Moher, A. Liberati, J. Tetzlaff, D.G. Altman, Preferred reporting items for systematic reviews and meta-analyses: the PRISMA statement, Int. J. Surg. 8 (5) (2010) 336-341 (PubMed PMID: 20171303. Epub 2010/02/23. eng).

[36] C.A. Lemos, V.E. de Souza Batista, D.A. Almeida, J.F. Santiago Junior, F.R. Verri, E.P. Pellizzer, Evaluation of cement-retained versus screw-retained implant-supported restorations for marginal bone loss: a systematic review and meta-analysis, J. Prosthet. Dent. 115 (4) (2016 Apr) 419-427 (PubMed PMID: 26589441. Epub 2015/11/22. eng).

[37] C.A. Lemos, C.C. Mello, D.M. dos Santos, F.R. Verri, M.C. Goiato, E.P. Pellizzer, Effects of platelet-rich plasma in association with bone grafts in maxillary sinus augmentation: a systematic review and meta-analysis, Int. J. Oral Maxillofac. Surg. 45 (4) (2016 Apr) 517-525 (PubMed PMID: 26775635. Epub 2016/01/19. eng)

[38] A.R. Jadad, R.A. Moore, D. Carroll, C. Jenkinson, D.J. Reynolds, D.J. Gavaghan, et al., Assessing the quality of reports of randomized clinical trials: is blinding necessary? Control. Clin. Trials 17 (1) (1996 Feb) 1-12 (PubMed PMID: 8721797. Epub 1996/ 02/01. eng).

[39] A. Halon, P. Donizy, M. Dziegala, R. Dobrakowski, K. Simon, Tissue laser biostimulation promotes post-extraction neoangiogenesis in HIV-infected patients, Lasers Med. Sci. 30 (2) (2015 Feb) 701-706 (PubMed PMID: 23917415. PMCID: PMC4320301. Epub 2013/08/07. eng).

[40] P. Vescovi, E. Merigo, M. Meleti, C. Fornaini, S. Nammour, M. Manfredi, Nd:YAG laser biostimulation of bisphosphonate-associated necrosis of the jawbone with and without surgical treatment, Br. J. Oral Maxillofac. Surg. 45 (8) (2007 Dec) 628-632 (PubMed PMID: 17524535. Epub 2007/05/26. eng).

[41] S. Oliveira Sierra, A. Melo Deana, R.A. Mesquita Ferrari, P. Maia Albarello, S.K. Bussadori, K.P. Santos Fernandes, Effect of low-level laser therapy on the post-surgical inflammatory process after third molar removal: study protocol for a doubleblind randomized controlled trial, Trials 14 (2013) 373 (PubMed PMID: 24195796. PMCID: PMC3832037. Epub 2013/11/08. eng).

[42] A.Z. Abd-Elaal, H.A. El-Mekawii, A.M. Saafan, L.A. El Gawad, Y.M. El-Hawary, M.A. Abdelrazik, Evaluation of the effect of low-level diode laser therapy applied during the bone consolidation period following mandibular distraction osteogenesis in the human, Int. J. Oral Maxillofac. Surg. 44 (8) (2015 Aug) 989-997 (PubMed PMID: 25979190. Epub 2015/05/17. eng).

[43] A. Dominguez, C. Gomez, J.C. Palma, Effects of low-level laser therapy on orthodontics: rate of tooth movement, pain, and release of RANKL and OPG in GCF, Lasers Med. Sci. 30 (2) (2015 Feb) 915-923 (PubMed PMID: 24346335. Epub 2013/12/ 19. eng).

[44] V.J. Garcia, J. Arnabat, R. Comesana, K. Kasem, J.M. Ustrell, S. Pasetto, et al., Effect of low-level laser therapy after rapid maxillary expansion: a clinical investigation, Lasers Med. Sci. 31 (6) (2016 Aug) 1185-1194 (PubMed PMID: 27236292. Epub 2016/ 05/30. eng).

[45] L. Eslamian, A. Borzabadi-Farahani, A. Hassanzadeh-Azhiri, M.R. Badiee, R. Fekrazad, The effect of 810-nm low-level laser therapy on pain caused by orthodontic elastomeric separators, Lasers Med. Sci. 29 (2) (2014 Mar) 559-564 (PubMed PMID: 23334785. Epub 2013/01/22. eng).

[46] M. Mozzati, G. Martinasso, N. Cocero, R. Pol, M. Maggiora, G. Muzio, et al., Superpulsed laser therapy on healing process after tooth extraction in patients waiting for liver transplantation, Lasers Med. Sci. 27 (2) (2012 Mar) 353-359 (PubMed PMID: 21279404. Epub 2011/02/01. eng).

[47] N. Angelov, S. Pesevska, M. Nakova, I. Gjorgoski, K. Ivanovski, D. Angelova, et al., Periodontal treatment with a low-level diode laser: clinical findings, Gen. Dent. 57 (5) (2009 Sep-Oct) 510-513 (PubMed PMID: 19903643. Epub 2009/11/12. eng).

[48] P. Chondros, D. Nikolidakis, N. Christodoulides, R. Rossler, N. Gutknecht, A. Sculean, Photodynamic therapy as adjunct to non-surgical periodontal treatment in patients on periodontal maintenance: a randomized controlled clinical trial, Lasers Med. Sci. 24 (5) (2009 Sep) 681-688 (PubMed PMID: 18465191. Epub 2008/05/10. eng).

[49] M. Youssef, S. Ashkar, E. Hamade, N. Gutknecht, F. Lampert, M. Mir, The effect of low-level laser therapy during orthodontic movement: a preliminary study, Lasers Med. Sci. 23 (1) (2008 Jan) 27-33 (PubMed PMID: 17361391. Epub 2007/03/16. eng).

[50] A.A. Zaky, H.M.M. El Shenawy, T.A.H. Harhsh, M. Shalash, N.M.I. Awad, Can low level laser therapy benefit bone regeneration in localized maxillary cystic defects? - a prospective randomized control trial, Maced. J. Med. Sci. 4 (4) (2016 Dec 15) 720-725.

[51] J.R. Landis, G.G. Koch, The measurement of observer agreement for categorical data, Biometrics 33 (1) (1977 Mar) 159-174 (PubMed PMID: 843571. Epub 1977/03/01. eng).

[52] A.C. Renno, P.A. McDonnell, N.A. Parizotto, E.L. Laakso, The effects of laser irradiation on osteoblast and osteosarcoma cell proliferation and differentiation in vitro, Photomed. Laser Surg. 25 (4) (2007 Aug) 275-280 (PubMed PMID: 17803384. Epub 2007/09/07. eng)

[53] A. Schindl, M. Schindl, H. Pernerstorfer-Schon, L. Schindl, Low-intensity laser therapy: a review, J. Investig. Med. 48 (5) (2000 Sep) 312-326 (PubMed PMID: 10979236. Epub 2000/09/09. eng).

[54] F.A. Al-Watban, X.Y. Zhang, B.L. Andres, Low-level laser therapy enhances wound healing in diabetic rats: a comparison of different lasers, Photomed. Laser Surg. 25 (2) (2007 Apr) 72-77 (PubMed PMID: 17508840. Epub 2007/05/19. eng). 